Pacific Journal of Mathematic

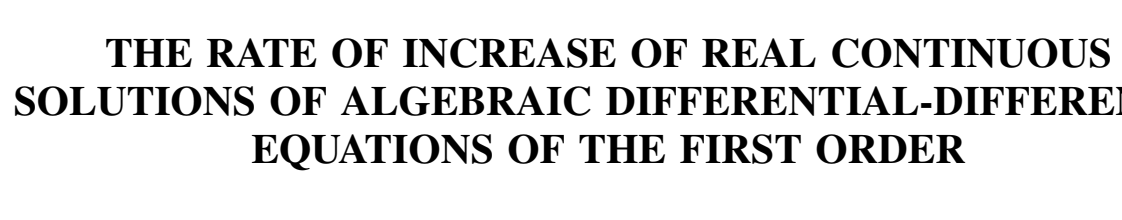




\title{
THE RATE OF INCREASE OF REAL CONTINUOUS SOLUTIONS OF ALGEBRAIC DIFFERENTIAL-DIFFERENCE EQUATIONS OF THE FIRST ORDER
}

\author{
K. L. COOKE
}

1. Introduction. It is the purpose of this paper to prove several theorems describing the rate of increase, as $t \longrightarrow+\infty$, of real solutions of algebraic differential-difference equations of the form

$$
P\left(t, u(t), u^{\prime}(t), u(t+1), u^{\prime}(t+1)\right)=0 .
$$

In this equation, and throughout this paper, $P(t, u, v, \cdots)$ denotes a polynomial in the variables $t, u, v, \cdots$, with real coefficients, and a prime denotes differentiation with respect to $t$. In order to explain the significance and limitations of these theorems, it is first necessary to summarize the work, by other investigators, which suggested the present discussion.

In 1899, E. Borel, [1], published a memoir in which he studied the magnitude of solutions of algebraic differential equations. His result, as later improved by E. Lindelöf, [4], is quoted here for reference:

Let $u(t)$ be a real function which is defined and which has a continuous first derivative for all $t$ larger than $t_{0}$, and which satisfies the first order algebraic differential equation

$$
P\left(t, u(t), u^{\prime}(t)\right)=0
$$

for $t>t_{0}$. Then there is a positive number $k$, which depends only on $P$, such that

$$
|u(t)|<\exp \left(t^{k / k}\right)
$$

for $t \geq t_{0}$.

It is noteworthy that it is impossible to prove a result of the above type for higher order equations. For a discussion of this point, refer to Vijayaraghavan, [ 7].

Received January 10, 1953.

Pacific J. Math. 4 (1954), 483 - 501 
Extensions of the Borel-Lindelöf method to difference equations have already been effected by Lancaster, [3], and Shah, [5] and [6]. Shah demonstrated that no theorem comparable to that of Borel and Lindelöf can be obtained for the class of algebraic difference equations of the form

$$
P(t, u(t), u(t+1))=0 .
$$

For, let $g(t)$ be an arbitrary increasing function which becomes indefinitely large as $t \rightarrow+\infty$. Shah proved that it is possible to construct an equation of the type (3) with a real solution $u(t)$ which exists and is continuous for $t \geq t_{0}$ and which exceeds $g(t)$ at each point of a sequence $\left\{t_{n}\right\}$ such that $t_{n} \rightarrow+\infty$ as $n \longrightarrow \infty$. The situation with respect to higher order equations is similar. Shah did, however, obtain the following weaker results concerning the possible rate of growth of solutions of ( 3 ):

There exists a positive number $A$, which depends only on the polynomial $P$, with the following property: if $u(t)$ is, for all $t \geq t_{0}$, a real continuous solution of ( 3 ), then there is no number $T$ such that ${ }^{1}$

$$
|u(t)|>e_{2}(A t) \quad \text { for all } t \geq T \text {. }
$$

That is, for each such solution $u(t)$ there is a sequence $t_{1}, t_{2}, \ldots\left(t_{n} \rightarrow+\infty\right.$ as $n \longrightarrow \infty$ ) such that

$$
|u(t)| \leq e_{2}(A t)
$$

for $t=t_{1}, t_{2}, \ldots$. If $u(t)$ is a real, continuous, monotonic solution of ( 3 ) for $t \geq t_{0}$, then there exists a number $\tau \geq t_{0}$ such that (4) holds for all $t \geq \tau$.

We shall now turn to a discussion of the class of differential-difference equations of the form ( 1 ). We first make the following definition.

DEFINITION. A real function $u(t)$ will be said to be a proper solution of a differential-difference equation (1) if there exists a number $t_{0}$ such that $u(t)$ exists and is a solution of (1) for all $t \geq t_{0}$, and such that $u(t)$ has a continuous first derivative for $t \geq t_{0}$.

In view of Shah's results on difference equations, it is not to be expected that a theorem analogous to the Borel-Lindelöf theorem should hold for first [2].

${ }^{1}$ We here employ the notation $e_{2}(x)=\exp \left(e^{x}\right)$ which was adopted by G. H. Hardy, 
order differential-difference equations. However, it might be expected that a result like that of Shah could be obtained for equations of the class (1). This is not the case, as is shown by the following theorem.

THEOREM 1. Let $g(t)$ be an arbitrary increasing function which becomes indefinitely large as $t \longrightarrow+\infty$. It is possible to construct an algebraic differential-difference equation of the form

$$
P\left(t, u(t), u^{\prime}(t), u(t+1), u^{\prime}(t+1)\right)=0
$$

which has a proper solution $u(t)$ which exceeds $g(t)$ for all $t$. This statement remains valid if equation $(1)$ is replaced by the equation

$$
P\left(t, u(t), u^{\prime}(t), u^{\prime}(t+1)\right)=0 \text {. }
$$

Proof. We shall prove this theorem at once by constructing a suitable example. Define a function $u(t)$ as follows. Let $u(t)=g(n+2)+1$ in the interval $[n, n+1]$, for $n=0,2,4, \ldots$ In the intervals $[n, n+1]$, where $n=1,3$, $5, \cdots$, let $u(t)$ be any continuous, non-decreasing function which has a continuous first derivative, and for which

$$
u(n)=g(n+1)+1, u(n+1)=g(n+3)+1, u^{\prime}(n)=u^{\prime}(n+1)=0 .
$$

It is clear that the function so defined satisfies the equation

$$
u^{\prime}(t) u^{\prime}(t+1)=0
$$

for all $t>0$. Furthermore, $u(t)$ is non-decreasing for all $t$ and $u(t)>g(t)$ for $t \geq 0$. Since equation (6) is in the class of equations of the form (1), and in the class of equations of the form (5), the proof of Theorem 1 is complete.

This theorem is in sharp contrast to those for algebraic differential or difference equations. It shows that no bound at all can be placed on the rate of growth of solutions of differential-difference equations of the form (1). The same difficulty intrudes even if we speak only of monotone solutions.

It is, however, possible to obtain useful bounds on the rate of growth of solutions of less general classes of differential-difference equations. We observe first of all that, according to Theorem 1 , no results like those of Borel or Shah can be obtained for the class of equations of the form (5). We shall, however, prove analogous results for equations of the following types:

$$
P\left(t, u(t), u^{\prime}(t+1)\right)=0
$$




$$
\begin{aligned}
& P\left(t, u(t), u^{\prime}(t), u(t+1)\right)=0 \\
& P\left(t, u^{\prime}(t), u(t+1)\right)=0 .
\end{aligned}
$$

Even for such equations it is not possible to establish a theorem like the BorelLindelöf theorem. This may be seen from the following simple counterexample. Let $g(t)$ be an arbitrary real, continuous, increasing function which becomes indefinitely large as $t \longrightarrow+\infty$. Let $m$ be any non-negative integer. Let $u(t)=t^{m}$ for $t$ in the intervals $[2 n, 2 n+1], n=0,1,2, \cdots$. For $t$ in the intervals $(2 n+1$, $2 n+2), n=0,1,2, \cdots$, let $u(t)$ be defined in any convenient fashion for which $u^{\prime}(t)$ is continuous and $u(2 n+3 / 2)>g(2 n+3 / 2)$. This function $u(t)$ exceeds $g(t)$ for arbitrarily large values of $t$, and satisfies each of the following equations for all $t>0$ :

$$
\begin{aligned}
& \left\{u^{\prime}(t+1)-m(t+1)^{m-1}\right\}\left\{u(t)-t^{m}\right\}=0 \\
& {\left[u(t+1)-(t+1)^{m}\right]\left[u^{\prime}(t)-m t^{m-1}\right]=0 .}
\end{aligned}
$$

Note that $(10)$ is an equation in the class (7) and equation (11) is in the class ( 8 ) and (9). Furthermore, all the above remarks are correct for $m=0$, in which case $(10)$ and (11) are equations with constant coefficients. The following theorem has therefore been proved.

THEOREM 2. Let $g(t)$ be an arbitrary increasing function which becomes indefinitely large as $t \rightarrow+\infty$. It is possible to construct a first order algebraic differential-difference equation of the form

$$
P\left(t, u(t), u^{\prime}(t+1)\right)=0
$$

with a proper solution $u(t)$ which exceeds $g(t)$ at each point of a sequence $\left\{t_{n}\right\}$ for which $t_{n} \longrightarrow+\infty$ as $n \rightarrow \infty$. The statement remains true if $(7)$ is replaced by equation ( 8 ) or equation (9), or by one of the equations

$$
\begin{aligned}
& P\left(u(t), u^{\prime}(t+1)\right)=0 \\
& P\left(u^{\prime}(t), u(t+1)\right)=0 .
\end{aligned}
$$

Although we cannot establish theorems of the Borel-Lindelof type for the classes of equations mentioned above, we have proved several results analogous to those of Shah. These results are stated in Theorems 3, 5, and 6 of $\$ 3$ below. Moreover, in Theorem 4, stated below, we have proved a theorem of the BorelLindelöf type for a certain subclass of equations of the type (7). No theorems 
are given in this paper for equations with higher order derivatives or differences, since results like those mentioned above can be obtained only for rather special classes of such equations.

2. Lemmas. In this section, we shall prove several lemmas which will be required in proving the theorems of 3 .

Lemma 1. Suppose that $u(t)$ is, for all $t \geq t_{0}$, a positive function with a continuous first derivative. Let $A$ and $B$ be two positive numbers for which $B<e^{A}$. Let $C$ be an arbitrary non-negative number. Suppose that there is a sequence $\left\{\tau_{n}\right\}$ for which $\tau_{n} \longrightarrow+\infty$ as $n \longrightarrow \infty$ and for which $u\left(\tau_{n}\right) \geq e_{2}\left(A \tau_{n}\right)$. Then there exists a sequence $\left\{t_{n}\right\}$ for which $t_{n} \longrightarrow+\infty$ as $n \rightarrow \infty$ and for which

$$
u^{\prime}\left(t_{n}+1\right)>t_{n}^{C} u\left(t_{n}\right)^{B} .
$$

Proof. Assume that $u(t)$ is a positive function with a continuous first derivative, and that

$$
u^{\prime}(t+1) \leq t^{C} u(t)^{B}
$$

for all $t \geq T$. We shall prove that as a consequence there is a number $T_{2}$ such that

$$
u(t)<e_{2}(A t)
$$

for $t \geq T_{2}$. This will prove Lemma 1 . We divide the proof of (15) into two cases.

Case 1. We assume that $B>1$. We may, of course, suppose that $T$ is as large as is convenient; choose $T$ so large that

$$
B^{j-1} \log T>j-1 \quad(j=1,2,3, \ldots) .
$$

This is certainly true for $j$ sufficiently large if $\log T>0$, and by choosing $T$ large enough we can ensure that it is true for all $j$. Then for $j=1,2,3, \ldots$,

$$
(2 T)^{B^{j-1}} \geq 2 T^{B^{j-1}}>T+e^{j-1} \geq T+j .
$$

Hlaving chosen $T$, define

$$
M^{\prime}=\max _{T \leq t \leq T+1} u(t), \quad M=\max \left(M^{\prime}, 1\right) .
$$

We shall now prove by induction that 


$$
u(t) \leq M^{B^{n}} \prod_{j=0}^{n}(T+j)^{(C+1) B^{n-j}}
$$

for $T+n \leq t \leq T+n+1(n=0,1,2, \ldots)$. This is evident for $n=0$. Suppose that (18) has been proved for $n=k-1(k \geq 1)$. Then by (18) and (14)

$$
u^{\prime}(t+1) \leq(1+k)^{C} M^{B^{k}} \prod_{j=0}^{k-1}(T+j)^{(C+1) B^{k-j}}
$$

for $T+k-1 \leq t \leq T+k$. Upon observing that the right hand side of inequality (18) is an increasing function of $n$, and employing (14) again, we get

$$
\begin{aligned}
u(T+k) & \leq u(T+1)+\int_{T}^{T+k-1} u^{\prime}(t+1) d t \\
& \leq M+(T+k)^{C}(k-1) M^{B} \prod_{j=0}^{k-1}(T+j)^{(C+1) B^{k-j}} \\
& \leq(T+k)^{C}(T+k-1) M^{B^{k}} \prod_{j=0}^{k-1}(T+j)^{(C+1) B^{k-j}} .
\end{aligned}
$$

On integrating the first inequality under (18) from $T+k-1$ to $t$, where $t \leq T+k$, and combining with the inequality just derived, we obtain

$$
u(t+1) \leq t(T+k)^{C} M^{B}{ }^{k} \prod_{j=0}^{k-1}(T+j)^{(C+1) B^{k-j}} \quad(T+k-1 \leq t \leq T+k) .
$$

Replacing $t$ by $T+k$ in the right member of the above inequality, we see that (18) is valid for $n=k$. This completes the inductive proof of (18).

We now employ (17). ( 18 ) takes the form $u(t) \leq\left[M(2 T)^{(n+1)(C+1)}\right]^{B^{n}}=e_{2}[n \log B+\log \{(1+n)(C+1) \log (2 T)+\log M\}]$ for $T+n \leq t \leq T+n+1$. Let $R=\max (2 T, M)$. Then

$$
u(t) \leq e_{2}[n \log B+\log (n C+n+C+2)+\log \log R]
$$

for $T+n \leq t \leq T+n+1$. Since $\log B<A$ by hypothesis, (15) follows. 
Case 2. We now assume that $B \leq 1$. Using the same method as in Case 1 , we can easily prove by induction that

$$
u(t) \leq M \prod_{j=0}^{n}(T+j)^{C+1}
$$

for $T+n \leq t \leq T+n+1(n=0,1,2, \cdots)$. Hence

$$
u(t) \leq M(T+n)^{(n+1)(C+1)} \leq M t^{(C+1)(t-T+1)}
$$

for $T+n \leq t \leq T+n+1$. (15) follows at once. This completes the proof of I emma 1 .

LEMMA 2. Suppose that $u(t)$ is, for all $t \geq t_{0}$, a positive non-decreasing function with a continuous first derivative, and that $u(t) \geq e_{2}(A t)$ for $t \geq t_{0}$. Let $B$ and $C$ be any non-negative numbers for which $B+C<e^{A}$, and let $D$ be

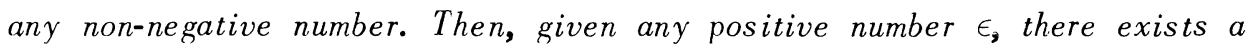
sequence $t_{1}, t_{2}, \cdots\left(t_{n} \longrightarrow+\infty\right.$ as $\left.n \longrightarrow \infty\right)$ such that

$$
\begin{aligned}
& u\left(t_{n}+1\right) \geq u\left(t_{n}\right)^{B} u^{\prime}\left(t_{n}\right)^{C} \\
& t_{n}^{D} u\left(t_{n}\right) \leq u^{\prime}\left(t_{n}\right) \leq u\left(t_{n}\right)^{1+\epsilon} \\
& (n=1,2, \cdots) \text {. }
\end{aligned}
$$

Proof. We divide the proof into two cases.

Case 1. Suppose that $u^{\prime}(t) \geq t^{D} u(t)$ for all sufficiently large $t$, say for $t \geq t_{0}$. It will be sufficient to prove the lemma for values of $\epsilon$ so small that $(B+C)(1+\epsilon)<e^{A}$. I et $\epsilon$ be any such number, and let $\alpha=(B+C)(1+\epsilon)$.

Borel, [1], proved that if a function $u(t)$ is, for all $t \geq t_{0}$, a positive, nondecreasing function with a continuous first derivative, then, given any positive number $\epsilon, u^{\prime}(t) \geq u(t)^{1+\epsilon}$ at most on a set of intervals the sum of whose lengths is a finite number (which depends on $\epsilon$ ). This result will hereafter be referred to as Borel's Lemma.

If $u(t)$ satisfies the hypotheses of Lemma 2, then by Borel's Lemma there is a number $T \geq t_{0}$ such that $u^{\prime}(t) \leq u(t)^{1+\epsilon}$ for all $t \geq T$, except for $t$ belonging to a set $E$ of intervals of total length less than $1 / 2$. We can now choose a number $\tau>T$ such that no point of the sequence $\tau, \tau+1, \tau+2, \cdots$, belongs to $E$. It follows that (21) holds for eact point $t_{n}=\tau+n$. We shall now show 
that $(20)$ holds at the points of an infinite subsequence of the sequence $\{\tau+n\}$. If this is not true, there is an integer $N$ such that

$$
u(\tau+n+1)<u(\tau+n)^{B} u^{\prime}(\tau+n)^{C}
$$
for all $n \geq N$.

This implies that

$$
u(\tau+n+1)<u(\tau+n)^{\alpha}
$$$$
\text { for } n \geq N \text {. }
$$

It follows that

$$
u(\tau+N+m)<e_{2}[m \log \alpha+\log \log u(\tau+N)]
$$

for $m=1,2,3, \ldots$. Since $\log \alpha<A$, this contradicts the hypothesis that $u(t) \geq e_{2}(A t)$ for $t \geq t_{0}$. It follows that there is an infinite subsequence of the sequence $\{\tau+n\}$ at which (20) is valid. This completes the proof in Case 1 .

Case 2. The alternative to the supposition of Case 1 is that $u^{\prime}(t)<t^{D} u(t)$ for arbitrarily large values of $t$. We define $\alpha$ as in Case 1 , and again suppose $\epsilon$ so small that $\log \alpha<A$. From the fact that $u(t) \geq e_{2}(A t)$ it follows that $u^{\prime}(t)>t^{D} u(t)$ for arbitrarily large $t$. By continuity of $u(t)$ and $u^{\prime}(t), u^{\prime}(t)<$ $t^{D} u(t)$ in open intervals, and $u^{\prime}(t) \geq t^{D} u(t)$ in closed intervals. Let the open intervals be

$$
\left(a_{1}, b_{1}\right),\left(a_{2}, b_{2}\right), \ldots,\left(a_{n}, b_{n}\right), \ldots
$$

$\left(a_{1} \geq t_{0}\right)$ and let the closed intervals be

$$
\left[b_{1}, a_{2}\right],\left[b_{2}, a_{3}\right], \ldots,\left[b_{n}, a_{n+1}\right], \ldots .
$$

Note that $a_{n} \longrightarrow+\infty$ and $b_{n} \longrightarrow+\infty$, and that

$$
u^{\prime}\left(a_{n}\right)=a_{n}^{D} u\left(a_{n}\right) \text { and } u^{\prime}\left(b_{n}\right)=b_{n}^{D} u\left(b_{n}\right) .
$$

By Borel's Lemma, $u^{\prime}(t) \leq u(t)^{1+\epsilon}$ for all $t$ except for $t$ in a set $E$ of open intervals of finite total length. Let $E_{n}$ be the subset of $E$ contained in $\left[b_{n}, a_{n+1}\right]$ and let $L_{n}$ be the sum of the lengths of the intervals of $E_{n}$. Then $\lim L_{n}=0$ as $n \rightarrow \infty$. We shall prove that there are arbitrarily large values of $n$ for which there is at least one point $t_{n}$ in the interval $\left[b_{n}, a_{n+1}\right]$ such that

$$
u\left(t_{n}+1\right) \geq u\left(t_{n}\right)^{B} u^{\prime}\left(t_{n}\right)^{C}
$$


and such that $t_{n}$ is not in $E_{n}$. The proof will be by contradiction. Assume the contrary. Then there is a positive integer $N$ such that, for every $n \geq N$,

$$
u(t+1)<u(t)^{B} u^{\prime}(t)^{C}
$$

for all $t$ which are in $\left[b_{n}, a_{n+1}\right]$ but not in $E_{n}$.

First we suppose that $0<a \leq 1$. Since $u(t) \geq e_{2}(A t)$, we may select an integer $p \geq N$ such that

$$
u\left(b_{n}\right)^{\epsilon}>b_{n}^{D}
$$
for all $n \geq p$.

Equations (22) therefore imply that

$$
u\left(b_{n}\right)^{1+\epsilon}>u^{\prime}\left(b_{n}\right)
$$

for all $n \geq p$.

Hence $b_{n}$ is not in $E_{n}$ if $n \geq p$. Consequently (23) implies that

$$
u\left(b_{p}+1\right)<u\left(b_{p}\right)^{B} u^{\prime}\left(b_{p}\right)^{C}<u\left(b_{p}\right)^{\alpha} \leq u\left(b_{p}\right) .
$$

But $u(t)$ is non-decreasing. Thus we have reached a contradiction, and (23) cannot be true if $0<\alpha \leq 1$.

Suppose, then, that $\alpha>1$. Just as before, we may select an integer $p \geq N$ such that $b_{n}$ is not in $E_{n}$ for $n \geq p$. We also choose $p$ so large that $L_{n}<1$ for $n \geq p$ and so large that

$$
\max _{\zeta \geq b_{p}} \frac{(D+1) \zeta^{D}}{\alpha^{\zeta} \log \alpha}<\alpha^{-b p} \log u\left(b_{p}\right)
$$

This is possible because the right-hand member becomes indefinitely large as $p \longrightarrow+\infty$, since $u(t) \geq e_{2}(A t)$ and $A>\log \alpha$, and because the maximum in the left member is finite. Define $c_{p}=b_{p}$. Wie shall now employ an inductive method to establish the existence of a sequence $c_{p}, c_{p}+1, c_{p}+2, \cdots$, for which

$$
\log u\left(c_{p}+i\right) \leq c_{0}{ }^{c}{ }_{p}+i-c_{p}+\sum \delta_{j} \log u\left(c_{p}\right)
$$

$(i=0,1,2, \cdots)$, where the summation is over all $j \geq p$ for which $b_{j} \leq c_{p+i-1}$, and where the $\delta_{j}$ are defined below. In the first place, it is clear that (25) is true for $i=0$. Suppose that we have established the existence of points $c_{p+1}$, $c_{p}+2, \cdots, c_{p+k-1}(k \geq 1)$ for which $(25)$ holds. There are now two possibilities: 
(a) One possibility is that the point $c_{p}+k-1$ lies in an interval $\left[b_{q}, a_{q+1}\right]$ for some value of $q$. If this is so, $c_{p+k-1}$ may lie in $E_{q}$, or it may not. Let $\epsilon_{q, 1}$ be the smallest non-negative number such that $c_{p+k-1}-\epsilon_{q, 1}$ is in $\left[b_{q}, a_{q+1}\right]$ but not in $E_{q}$. Such a number exists, since $b_{q}$ is not in $E_{q}$. Then, by (23),

$$
u\left(c_{p+k-1}+1-\epsilon_{q, 1}\right)<u\left(c_{p+k-1}-\epsilon_{q, 1}\right)^{B} u^{\prime}\left(c_{p+k-1}-\epsilon_{q, 1}\right)^{C} .
$$

By Borel's Lemma and the fact that $u(t)$ is non-decreasing, this gives rise to

$$
u\left(c_{p+k-1}+1-\epsilon_{q, 1}\right)<u\left(c_{p+k-1}\right)^{a} .
$$

Since $\epsilon_{q, 1}<L_{q}<1$, the points $c_{p+k-1}$ and $c_{p}+k_{-1}+1-\epsilon_{q, 1}$ cannot lie in the same interval of $E_{q}$. If $c_{p+k-1}+1-\epsilon_{q, 1}>a_{q+1}$, we define $c_{p}+k=c_{p}+k-1+$ $1-\epsilon_{q, 1}$. If not, we proceed as follows. Let $\epsilon_{q, 2}$ be the smallest non-negative number such that $c_{p+k-1}+1-\epsilon_{q, 1}-\epsilon_{q, 2}$ is in $\left[b_{q}, a_{q+1}\right]$ but not in $E_{q}$. Using (23) again, we find that

$$
u\left(c_{p+k-1}+2-\epsilon_{q, 1}-\epsilon_{q, 2}\right)<u\left(c_{p+k-1}+1-\epsilon_{q, 1}-\epsilon_{q, 2}\right)^{\alpha}
$$

and therefore that

$$
\log u\left(c_{p+k-1}+2-\epsilon_{q, 1}-\epsilon_{q, 2}\right)<\alpha^{2} \log u\left(c_{p+k-1}\right) .
$$

We continue in this manner, obtaining a sequence of points

$$
c_{p+k-1}, c_{p+k-1}+1-\epsilon_{q, 1}, c_{p+k-1}+2-\epsilon_{q, 1}-\epsilon_{q, 2}, \cdots,
$$

no two of which can lie in the same interval of $E_{q}$. Let

$$
c_{p+k-1}+r-\epsilon_{q, 1}-\cdots-\epsilon_{q, r}
$$

be the first point in this sequence which is larger than $a_{q+1}$; such a point must .exist since

$$
\delta_{q}=\epsilon_{q, 1}+\epsilon_{q, 2}+\cdots+\epsilon_{q, r} \leq L_{q}<1
$$

Define

$$
c_{p+k}=c_{p+k-1}+r-\epsilon_{q, 1}-\cdots-\epsilon_{q, r} \cdot
$$

Then 


$$
\log u\left(c_{p+k}\right)<\alpha^{r} \log u\left(c_{p+k-1}\right)=\alpha^{c}{ }^{c}+k-c_{p}+k-1+\delta_{q} \log u\left(c_{p}+k-1\right) .
$$

Combining this result with (25) for $i=k-1$, we find that (25) holds for $i=k$, with the choice of $c_{p}+k$ made above.

(b) The alternative to $(\mathrm{a})$ is that $c_{p}+k-1$ lies in an interval $\left(a_{q}, b_{q}\right)$ for some value of $q$. (In this case $k \geq 2$, since $c_{p}=b_{p}$.) Now $u^{\prime}(t)<t^{D} u(t)$ for all $t$ in this interval. Hence, by integration,

$$
u\left(b_{q}\right)<u\left(c_{p+k-1}\right) \exp \left(b_{q}^{D+1}-c_{p+k-1}^{D+1}\right)
$$

In this case, we define $c_{p+k}=b_{q}$. We shall now show that, with this choice of $c_{p}+k,(25)$ is satisfied for $i=k$. From the extended theorem of the mean and the inequality (24) we can deduce

$$
c_{p+k}^{D+1}-c_{p+k-1}^{D+1}<\left(\alpha^{c p+k}-\alpha^{c_{p}+k-1}\right) \alpha^{-c} p \log u\left(c_{p}\right) .
$$

This inequality will still be true if, in the right member, we place the additional factor

$$
a^{\sum \delta_{j}}
$$

where the summation is over all $j \geq p$ for which $b_{j} \leq c_{p}+k-2$. Using this result, (26), and (25) for $i=k-1$, we obtain

$$
\log u\left(c_{p+k}\right)<c_{p+k}^{D+1}-c_{p+k-1}^{D+1}+\log u\left(c_{p+k-1}\right)<\alpha^{c_{p}+k}-c_{p}+\sum_{\delta_{j}} \log u\left(c_{p}\right) .
$$

The inequality (25) for $i=k$ is an immediate consequence.

This completes the proof that there is a sequence of points $c_{p}+i$ for which (25) is valid. It is clear that $c_{p}+i \rightarrow+\infty$ as $i \rightarrow \infty$. Since the sum of the $\delta_{j}(j=1,2, \ldots)$ is no greater than the sum $L$ of the lengths of all the intervals of $E$,

$$
\log u(t) \leq a^{t-c_{p}+L} \log u\left(c_{p}\right)=\exp \left[\left(t-c_{p}+L\right) \log \alpha+\log \log u\left(c_{p}\right)\right]
$$

for $t=c_{p+i}(i=0,1,2, \cdots)$. Since $\log a<A$, it is a consequence of the above inequality that there is a positive integer $I$ such that $\log u(t)<\exp (A t)$ for $t=c_{p}+i(i \geq I)$. This contradicts the hypothesis of Lemma 2. Therefore (23) cannot be true if $\alpha>1$. Hence, no matter what the value of $\alpha$, there are arbitrarily large values of $n$ for which there is at least one point $t_{n}$ in the interval 
$\left[b_{n}, a_{n+1}\right]$ such that

$$
u\left(t_{n}+1\right) \geq u\left(t_{n}\right)^{B} u^{\cdot}\left(t_{n}\right)^{C},
$$

and such that $t_{n}$ is not in $E_{n}$. Since $t_{n}$ is not in $E_{n}, u^{\prime}\left(t_{n}\right) \leq u\left(t_{n}\right)^{1+\epsilon}$ for each such $t_{n}$. Since $t_{n}$ lies in $\left[b_{n}, a_{n+1}\right]$, we have $u^{\prime}\left(t_{n}\right) \geq t_{n}^{D} u\left(t_{n}\right)$. This completes the proof of the lemma.

Lemma 3. Suppose that $u(t)$ is, for all $t \geq t_{0}$, a positive funciion with a continuous first derivative, and that $u(t) \geq e_{2}(A t)$ for all $t \geq t_{0}$. Let $C$ be any non-negative number less than $e^{A}$. Then there is a sequence $t_{1}, t_{2}, \ldots$ $\left(t_{n} \longrightarrow+\infty\right.$ as $\left.n \longrightarrow \infty\right)$ such that

$$
u\left(t_{n}+1\right) \geq u^{\prime}\left(t_{n}\right)^{C}, \quad u^{\prime}\left(t_{n}\right) \geq e^{t_{n}} .
$$

Proof. First we suppose that there is a number $T \geq t_{0}$ such that $u^{\prime}(t) \geq e^{t}$ for $t \geq T$. Then $u(t)$ is non-decreasing for $t \geq T$, and the result follows at once from Lemma 2.

On the other hand, suppose that $u^{\prime}(t)<e^{t}$ for arbitrarily large values of $t$. Since $u(t) \geq e_{2}(A t), u^{\prime}(t)>e^{t}$ for arbitrarily large values of $t$. Therefore there is a sequence of numbers $t_{1}, t_{2}, \ldots\left(t_{n} \rightarrow+\infty\right.$ as $\left.n \rightarrow \infty\right)$ such that $u^{\prime}\left(t_{n}\right)=\exp \left(t_{n}\right)$. There exists a positive integer $N$ such that

$$
u\left(t_{n}+1\right) \geq e_{2}\left\{A\left(t_{n}+1\right)\right\}>\left(e^{t_{n}}\right)^{C}=u^{\prime}\left(t_{n}\right)^{C}
$$

for $n \geq N$. This completes the proof of Lemma 3 .

3. Theorems. We can now state and prove the theorems alluded to in the last paragraph of the introduction. The first of these is the following.

THEOREM 3. Consider any equation of the form

$$
P\left(t, u(t), u^{\prime}(t+1)\right)=0 .
$$

There exists a positive number $A$, which depends only on the polynomial $P$, with the following property: to each proper solution $u(t)$ of $(7)$ there corresponds a sequence $t_{1}, t_{2}, \ldots\left(t_{n} \longrightarrow+\infty\right.$ as $\left.n \longrightarrow \infty\right)$ such that

$$
|u(t)|<e_{2}(A t)
$$

for $t=t_{n}(n=1,2,3, \ldots)$. That is, if $u(t)$ is a proper solution of. $(7)$ then there 
is no number $T>0$ for which $|u(t)| \geq e_{2}(A t)$ for all $t \geq T$.

Proof. Equation (7) may be written in the form

$$
\sum_{i=0}^{I} \sum_{j=0}^{J} \sum_{k=0}^{K} T_{i j k}=0
$$

where

$$
T_{i j k}=a_{i j k} t^{i} u(t)^{j} u^{\prime}(t+1)^{k} .
$$

The $a_{i j k}$ are real numbers independent of $t$. Among the terms $T_{i j k}$ there is one term $T_{p q r}$ selected in the following way:

(1) Choose $r=K$.

(2) Choose $q$ to be the greatest of the values of $j$ among all the terms $T_{i j r}$.

(3) Choose $p$ to be the greatest of the values of $i$ among all the terms $T_{i q r}$. The term $T_{p q r}$ so defined will be called the principal term.

Except for constant factors, the ratios $T_{i j k} / T_{p q r}$ are of the following three possible types (excluding the ratio $T_{p q r} / T_{p q r}$ ):

$$
\left\{\frac{t^{r_{0}} u(t)^{r_{1}}}{u^{\prime}(t+1)}\right\}^{r-k}
$$

where $r_{0}$ and $r_{1}$ are rational numbers and $r>k$.

$$
\left\{\frac{t^{r_{2}}}{u(t)}\right\}^{q-j}
$$

where $r_{2}$ is a rational number and $q>j$.

where $p>i$. Let $R$ be the least non-negative number which is greater than or equal to the maximum value of $r_{1}$ for all ratios of type (a). Let $A$ be any positive number such that $e^{A}>R$.

Now suppose that $u(t)$ is a proper solution of (7) and that $u(t) \geq e_{2}(A t)$ for $t \geq T$. Choose $B$ so that $R<B<e^{A}$. It follows from Lemma 1 that there exists a sequence $\left\{t_{n}\right\}$ for which $t_{n} \longrightarrow+\infty$ as $n \longrightarrow \infty$ and for which $u^{\prime}\left(t_{n}+1\right)>$ $u\left(t_{n}\right)^{B}$. For each value $t=t_{n}$, the function $u(t)$ satisfies not only equation 
( 7), but also the equation

$$
\sum_{i=0}^{I} \sum_{j=0}^{J} \sum_{k=0}^{K} \frac{T_{i j k}}{T_{p q r}}=0
$$

Since $u(t) \geq e_{2}(A t)$, all ratios of types (b) or (c) approach zero as $t_{n} \longrightarrow+\infty$. Each ratio of type $(a)$ is bounded by

$$
\left\{\frac{t^{r_{0}} u(t)^{R}}{u(t)^{B}}\right\}^{r-k}
$$

when $t=t_{n}$, for appropriate values of $r_{0}$ and $k$. Since $B>R$ and $r>k$, each such ratio approaches zero on the sequence $\left\{t_{n}\right\}$. It now follows that we may find a positive integer $N$ such that the sum of all ratios $T_{i j k} / T_{p q r}$ is less than one in absolute value when $t=t_{N}$, whereas $T_{p q r} / T_{p q r}=1$. Thus (28) cannot be satisfied at the point $t_{N}$. This contradiction shows that a proper solution $u(t)$ of (7) cannot satisfy $u(t) \geq e_{2}(A t)$ for all $t \geq T$.

Moreover, a proper solution $u(t)$ of (7) cannot satisfy $u(t) \leq-e_{2}(A t)$ for all $t \geq T$. For if it could, the function $U(t)=-u(t)$ would satisfy $U(t) \geq$ $e_{2}(A t)$ for $t \geq T$ and would be a proper solution of an equation of the type (7). We have just shown that this is impossible. Since a proper solution is continuous, this completes the proof of Theorem 3.

The following theorem gives a much stronger result than does Theorem 3 , but for a smaller class of equations.

THE OREM 4. Let $u(t)$ be a non-decreasing or non-increasing proper solution of an equation of the form

$$
\sum_{i=0}^{I} a_{i L K} t^{i} u(t)^{L} u^{\prime}(t+1)^{K}+\sum_{i, j, k} a_{i j k} t^{i} u(t)^{j} u^{\prime}(t+1)^{k}=0
$$

wherein the $a_{i j k}$ are constants and the latter summation is a triple summation over the ranges $i=0,1, \ldots, I ; j=0,1, \ldots, J ; k=0,1, \cdots, K-1$. ( $L$ may be greater than $J$, equal to $J$, or less than J.) Then there exists a number $A>0$, which depends only on the form of (29), and there exists a number $T>0$, which depends on (29) and on $u(t)$, such that

$$
|u(t)|<e_{2}(A t)
$$


for all $t \geq T$

Proof. The method used in the proof of Theorem 3 for selecting the principal term $T_{p q r}$ leads to the choice $p=I, q=L, r=K$ for the equation (29). Except for constant factors, the ratios $T_{i j k} / T_{p q r}$ are of the following two possible types (excluding the ratio $T_{p q r} / T_{p q r}$ ):

$$
\left\{\frac{t^{r_{0}} u(t)^{r_{1}}}{u^{\prime}(t+1)}\right\}^{K-k}
$$

where $r_{0}$ and $r_{1}$ are rational and $K>k$.

$$
t^{i-1}
$$

where $I>i$. Define $R, A$, and $B$ as in the proof of Theorem 3. Let $C$ be any positive number for which $C / 2$ is larger than the maximum value of $r_{0}$ for all ratios of type (a).

Now suppose that $u(t)$ is a proper, non-decreasing solution of (29) for which $u(t) \geq e_{2}(A t)$ for a sequence $\left\{\tau_{n}\right\}$ of values of $t$ for which $\tau_{n} \longrightarrow+\infty$ as $n \rightarrow \infty$. It follows from Lemma 1 that there exists a sequence $\left\{t_{n}\right\}$ for which $t_{n} \longrightarrow+\infty$ and for which $u^{\prime}\left(t_{n}+1\right)>t_{n}^{C} u\left(t_{n}\right)^{B}$. For each value $t=t_{n}$, the function $u(t)$ satisfies not only equation (29), but also the equation (28) obtained by dividing by the principal term. But for $t=t_{n}$ all ratios of type (b) approach zero as $n \longrightarrow \infty$. Each ratio of type $(\mathrm{a})$ is bounded by

$$
\left\{\frac{t^{C / 2} u(t)^{R}}{t^{C} u(t)^{B}}\right\}^{K-k} .
$$

Since $B>R$ and $K>k$, and since $u\left(t_{n}\right) \rightarrow+\infty$ as $t_{n} \rightarrow+\infty$, each such ratio approaches zero. We thus obtain the same contradiction as in the proof of Theorem 3. No such solution $u(t)$ can exist. Therefore to each proper nondecreasing solution $u(t)$ there corresponds a $T>0$ such that $|u(t)|<e_{2}(A t)$ for all $t \geq T$.

If a proper, non-increasing solution $u(t)$ exists for which $u(t) \leq-e_{2}(A t)$ for $t=\tau_{n}(n=1,2, \cdots)$, where $\tau_{n} \longrightarrow+\infty$ as $n \rightarrow \infty$, we define $U(t)=-u(t)$, and obtain the same contradiction. Therefore to each proper, non-increasing solution $u(t)$ there corresponds a $T>0$ such that $|u(t)|<e_{2}(A t)$ for all $t \geq T$. This completes the proof of Theorem 4 .

Our next theorem is as follows. 
THEOREM 5. Consider any equation of the form

$$
P\left(t, u(t), u^{\prime}(t), u(t+1)\right)=0 \text {. }
$$

There exists a positive number $A$, which depends only on the polynomial $P$, with the following property: to each proper non-decreasing or non-increasing solution $u(t)$ of (8) there corresponds a sequence $t_{1}, t_{2}, \cdots\left(t_{n} \rightarrow+\infty\right.$ as $n \longrightarrow \infty)$ such that

$$
|u(t)|<e_{2}(A t)
$$

for $t=t_{n}(n=1,2, \ldots)$. That is, if $u(t)$ is any proper non-decreasing or nonincreasing solution of (8), there is no number $T>0$ for which $|u(t)| \geq e_{2}\left(A_{t}\right)$ for all $t \geq T$.

Proof. Equation ( 8) may be written in the form

$$
\sum_{h=0}^{H} \sum_{i=0}^{I} \sum_{j=0}^{J} \sum_{k=0}^{K} T_{h i j k}=0
$$

where

$$
T_{h i j k}=a_{h i j k} t^{h} u(t)^{i} u^{\prime}(t)^{j} u(t+1)^{k} .
$$

The $a_{h i j k}$ are real numbers independent of $t$. We select a principal term $T_{p q r s}$ in the following way. Let $S$ be the set of all terms $T_{h i j k}$. Let $S_{1}$ be the subset of $S$ consisting of those terms for which $k=K$. Let $M_{1}$ be the maximum value of $i+j$ for all terms in $S_{1}$. Let $S_{2}$ be the set consisting of those terms of $S_{1}$ for which $i+j=M_{1}$. Let $M_{2}$ be the maximum value of $j$ for all terms in $S_{2}$. Let $S_{3}$ be the set containing those terms of $S_{2}$ for which $j=M_{2}$. Let $M_{3}$ be the maximum value of $h$ for all terms in $S_{3}$. There is a unique term in $S_{3}$ for which $h=M_{3}$. This term will be called the principal term. We shall use the symbol $T_{\text {pqrs }}$ for it.

Except for constant factors, the ratios $T_{h i j k} / T_{p q r s}$ are of the following possible types (excluding the ratio $T_{p q r s} / T_{p q r s}$ ):

$$
\left\{\frac{t^{r_{0}} u(t)^{r_{1}} u^{\prime}(t)^{r_{2}}}{u(t+1)}\right\}^{s-k}
$$

where $r_{0}, r_{1}$, and $r_{2}$ are rational numbers and $s>k$. 


$$
t^{h-p} u(t)^{i-q} u^{\prime}(t)^{j-r}
$$

where $q+r>i+j$. Since $i, j, q$, and $r$ are integers, terms of type (b) fall into one of the following two sub-classes.

$$
\frac{t^{h-p} u(t)^{m}}{u^{\prime}(t)^{m+n}}
$$

where $m$ is an integer, $h-p$ is an integer, $n$ is a positive integer, and $m+n$ is a non-negative integer.

$$
\frac{t^{h-p} u^{\prime}(t)^{m}}{u(t)^{m+n}}=\left\{\frac{t^{r_{3}} u^{\prime}(t)}{u(t)^{1+r_{4}}}\right\}^{m}
$$

where $m$ and $n$ are positive integers, $h-p$ is an integer, $r_{3}$ is a rational number, and $r_{4}$ is a positive rational number.

$$
\left\{\frac{t^{r_{5}} u(t)}{u^{\prime}(t)}\right\}^{r-j}
$$

where $r_{5}$ is a rational number and $r>j$.

$$
t^{h-p}
$$

where $p>h$.

Let $R_{0}$ be the maximum value of $r_{0}$ for all ratios of type (a). Let $R_{1}^{\prime}$ be the maximum value of $r_{1}$ for all ratios of type $(\mathrm{a})$, and let $R_{1}=\max \left(0, R_{1}^{\prime}\right)$. Let $R_{2}^{\prime}$ be the maximum value of $r_{2}$ for all ratios of type (a), and let $R_{2}=\max$ $\left(0, R_{2}^{\prime}\right)$. Let $A$ be any number such that $e^{A}>R_{1}+R_{2}$. Select any numbers $B$ and $C$ for which $B>R_{1}, C>R_{2}$, and $B+C<e^{A}$. Let $R_{3}$ be the maximum value of $r_{3}$ and let $M$ be the maximum value of $m$ for all ratios of type (b2). Let $R_{4}$ be the minimum value of $r_{4}$ for all ratios of type (b2). Let $\epsilon$ be any positive number less than $R_{4} / 2$. Let $R_{5}^{\prime}$ be the maximum value of $r_{5}$ for all ratios of type (c), and let $R_{5}=\max \left(0, R_{5}^{\prime}\right)$. Select any number $D$ for which $D>R_{5}$.

Now assume that there exists a proper, non-decreasing solution $n(t)$ of (8) for which $u(t) \geq e_{2}(A t)$ for all $t \geq t_{0}$. By Lemma 2 there exists a sequence $\left\{t_{n}\right\}$ such that $(20)$ and $(21)$ are satisfied. For each value $t=t_{n}, u(t)$ satisfies not only equation ( 8$)$, but also the equation 


$$
\sum_{h=0}^{H} \sum_{i=0}^{I} \sum_{j=0}^{J} \sum_{k=0}^{K} \frac{T_{h i j k}}{T_{p q r s}}=0 .
$$

Now since $u(t) \geq e_{2}(A t)$ and $u^{\prime}(t) \geq t^{D} u(t)$ when $t=t_{n}$, all ratios of types (bl), (c), and (d) approach zero as $t_{n} \longrightarrow+\infty$. Also, each ratio of type (a) is, according to $(20)$, bounded by

$$
\left\{\frac{t^{R_{0}} u(t)^{R_{1}} u^{\prime}(t)^{R_{2}}}{u(t)^{B} u^{\prime}(t)^{C}}\right\}^{s-k}
$$

when $t=t_{n}$; and each ratio of type (b2) is, according to ( 21$)$, bounded by

$$
\left\{\frac{t^{R_{3}} u^{\prime}(t)}{u(t)^{1+2 \epsilon}}\right\}^{M}<\left\{\frac{t^{R_{3}} u^{\prime}(t)}{u^{\prime}(t) u(t)^{\epsilon}}\right\}^{M}
$$

when $t=t_{n}$. Since $B>R_{1}$ and $C>R_{2}$, all these ratios tend to zero as $t_{n} \longrightarrow+\infty$. This conclusion yields a contradiction, just as in the proofs of the earlier theorems. Therefore no such solution $u(t)$ can exist.

The assumption that a proper, non-increasing solution $u(t)$ satisfies $u(t) \leq$ $-e_{2}(A t)$ for all $t \geq t_{0}$ may be shown to lead to a contradiction by defining $U(t)=-u(t)$.

The conclusion stated in Theorem 5 follows.

Our final theorem is the following.

TheOREM 6. Consider any equation of the form

$$
P\left(t, u^{\prime}(t), u(t+1)\right)=0 .
$$

There exists a positive number $A$, which depends only on the polynomial $P$, with the following property: to each proper solution $u(t)$ of $(9)$ there corresponds a sequence $t_{1}, t_{2}, \cdots\left(t_{n} \longrightarrow+\infty\right.$ as $\left.n \rightarrow \infty\right)$ such that

$$
|u(t)|<e_{2}(A t)
$$

for $t=t_{n}(n=1,2, \ldots)$. That is, if $u(t)$ is any proper solution of $(9)$, there is no positive number $T$ for which $|u(t)| \geq e_{2}($ At $)$ for all $t \geq T$.

Proof. Equation (9) may be written in the form (27), where

$$
T_{i j k}=a_{i j k} t^{i} u^{\prime}(t)^{j} u(t+1)^{k} .
$$


The principal term $T_{p q r}$ is selected as follows:

(1) $r=K$;

(2) $q$ is the greatest of the values of $j$ among all terms $T_{i j r}$;

(3) $p$ is the greatest of the values of $i$ among all terms $T_{i q r}$.

By using Lemma 3, the proof of Theorem 6 may now be completed in much the same way as before. We omit the details.

\section{REFERENCES}

1. E. Borel, Mémoire sur les séries divergentes, Annales de l'Ecole Normale Superieure, 16 (1899), 9-136.

2. G. H. Hardy, Orders of infinity, Cambridge Tract No. 12, Cambridge University Press, 1910.

3. O. E. Lancaster, Some results concerning the behavior at infinity of real continuous solutions of algebraic difference equations, Bull. Amer. Math. Soc., 46 (1940), $169-177$.

4. E. Lindelöf, Sur la croissance des intégrales des équations différentielles algébriques du premier ordre, Bull. Soc. Math. France, 27 (1899), $205-215$.

5. S. M. Shah, On real continuous solutions of algebraic difference equations, Bull. Amer. Math. Soc., 53 (1947), 548-558.

6. - On real continuous solutions of algebraic difference equations, II, Proc. National Inst. Sci. India 16 (1950), $11-17$.

7. T. Vijayaraghavan, N.M. Basu, and S. N. Bose, A simple example for a theorem of Vijayaraghavan, Jour. London Math. Soc., 12 (1937), 250-252.

\section{Washington State College}





\section{PACIFIC JOURNAL OF MATHEMATICS}

\section{EDITORS}

\author{
M.M. SCHIFFER * \\ Stanford University \\ Stanford, California \\ E. HEWITT \\ University of Washington \\ Seattle 5, Washington
}

\section{R.P. DILWORTH}

California Institute of Technology Pasadena 4, California

\section{E. F. BECKENBACH**}

University of California

Los Angeles 24, California

\section{ASSOCIATE EDITORS}

$\begin{array}{llll}\text { H. BUSEMANN } & \text { P.R. HALMOS } & \text { BØRGE JESSEN } & \text { J. J. STOKER } \\ \text { HERBERT FEDERER } & \text { HEINZ HOPF } & \text { PAUL LÉVY } & \text { E.G. STRAUS } \\ \text { MARSHALL HALL } & \text { R.D. JAMES } & \text { GEORGE PÓLYA } & \text { KÔSAKU YOSIDA }\end{array}$

\section{SPONSORS}

UNIVERSITY OF BRITISH COLUMBIA

CALIFORNIA INSTITUTE OF TECHNOLOGY

UNIVERSITY OF CALIFORNIA, BERKELEY

UNIVERSITY OF CALIFORNIA, DAVIS

UNIVERSITY OF CALIFORNIA, LOS ANGELES

UNIVERSITY OF CALIFORNIA, SANTA BARBARA

UNIVERSITY OF NEVADA

OREGON STATE COLLEGE

UNIVERSITY OF OREGON
UNIVERSITY OF SOUTHERN CALIFORNIA STANFORD RESEARCH INSTITUTE STANFORD UNIVERSITY WASHINGTON STATE COLLEGE UNIVERSITY OF WASHINGTON

AMERICAN MATHEMATICAL SOCIETY HUGHES AIRCRAFT COMPANY

Mathematical papers intended for publication in the Pacific Journal of Mathematics should be typewritten (double spaced), and the author should keep a complete copy. Manuscripts may be sent to any of the editors. Manuscripts intended for the outgoing editors should be sent to their successors. All other communications to the editors should be addressed to the managing editor, E.G. Straus, at the University of California Los Angeles 24, California.

50 reprints of each article are furnished free of charge; additional copies may be obtained at cost in multiples of 50 .

The Pacific Journal of Mathematics is published quarterly, in March, June, September, and December. The price per volume (4 numbers) is $\$ 12.00$; single issues, $\$ 3.50$; back numbers (Volumes $1,2,3$ ) are available at $\$ 2.50$ per copy. Special price to individual faculty members of supporting institutions and to individual members of the American Mathematical Society: $\$ 4.00$ per volume; single issues, $\$ 1.25$.

Subscriptions, orders for back numbers, and changes of address should be sent to the publishers, University of California Press, ,Berkeley 4, California.

Printed at Ann Arbor, Michigan. Entered as second class matter at the Post Office, Berkeley, California.

* To be succeeded in 1955, by H.L. Royden, Stanford University, Stanford, California.

** To be succeeded in 1955, by E.G. Straus, University of California, Los Angeles 24, Calif.

UNIVERSITY OF CALIFORNIA PRESS - BERKELEY AND LOS ANGELES 


\section{Pacific Journal of Mathematics}

\section{Vol. 4, No. $4 \quad$ August, 1954}

Paul Civin, Orthonormal cyclic groups .................... 481

Kenneth Lloyd Cooke, The rate of increase of real continuous solutions of

algebraic differential-difference equations of the first order ......... 483

Philip J. Davis, Linear functional equations and interpolation series . . . . . 503

F. Herzog and G. Piranian, Sets of radial continuity of analytic functions . . 533

P. C. Rosenbloom, Comments on the preceding paper by Herzog and

Piranian ............................................. 539

Donald G. Higman, Remarks on splitting extensions .............. 545

Margaret Jackson, Transformations of series of the type ${ }_{3} \Psi_{3} \ldots \ldots \ldots \ldots 557$

Herman Rubin and Patrick Colonel Suppes, Transformations of systems of relativistic particle mechanics ....................... 563

A. Seidenberg, On the dimension theory of rings. II .............. 603

Bertram Yood, Difference algebras of linear transformations on a Banach

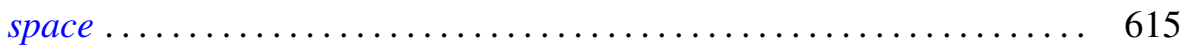

